18. Finkel R, Day J, Darras B, et al. Phase 1 study of intrathecal administration of AVXS-101 gene-replacement therapy (GRT) for spinal muscular atrophy type 2 (SMA2) (STRONG). Presented at: AAN 2019 Annual Meeting; May 4-10, 2019; Philadelphia, PA.

19. Mannaa MM, Kalra M, Wong B, Cohen AP, Amin RS. Survival probabilities of patients with childhood spinal muscle atrophy. J Clin Neuromuscul Dis. 2009;10(3):85-89.
20. Oskoui M, Levy G, Garland CJ, et al. The changing natural history of spinal muscular atrophy type 1. Neurology. 2007;69(20):1931-36.

21. Finkel RS, McDermott MP, Kaufmann P, et al. Observational study of spinal muscular atrophy type I and implications for clinical trials. Neurology. 2014;83(9):810-17.

22. Glascock J, Sampson J, Haidet-Phillips A, et al. Treatment algorithm for infants diagnosed with spinal muscular atrophy through newborn screening. J Neuromuscul Dis. 2018;5(2):145-58.

\title{
Spinal Muscular Atrophy Therapies: ICER Grounds the Price to Value Conversation in Facts
}

\author{
Catherine I. Starner, PharmD, and Patrick P. Gleason, PharmD
}

\section{COMMENTARY} for Clinical and Economic Review (ICER) released its final evidence report on the effectiveness and value of spinal muscular atrophy (SMA) treatments. ${ }^{1}$ The 2 SMA treatments approved by the U.S Food and Drug Administration were the focus of the report. Spinraza (nusinersen) is a survival motor neuron-2-directed antisense oligonucleotide indicated for SMA in pediatric and adult patients. ${ }^{2}$ Zolgensma (onasemnogene abeparvovec-xioi) is a 1-time gene therapy infusion indicated for SMA in children aged $<2$ years with bi-allelic mutations in the survival motor neuron 1 (i.e., SMA type 1) gene. ${ }^{3}$ Zolgensma has been dubbed the "most expensive drug in the world" at $\$ 2.125$ million. ${ }^{4}$ The ICER quality-adjusted life-year (QALY) costeffective model for treating SMA type 1 infants with symptoms who are $<8$ months found that Zolgensma should be priced at $\$ 310,000$ for a $\$ 100,000$ investment per QALY gained, 6.9-fold

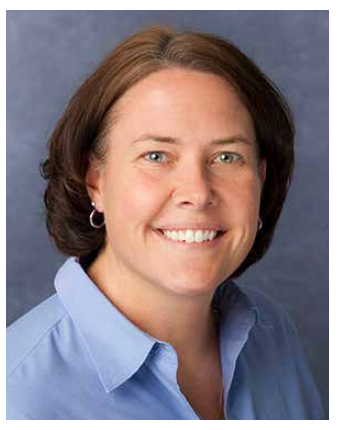
investment point of $\$ 100,000$ per QALY. Therefore, the Spinraza current price should be discounted by 10 -fold for a fair price at an investment point of $\$ 100,000$ per QALY gained.

Why are the cost-effective numbers so different for Spinraza and Zolgensma? The Spinraza value price is maximally $\$ 36,400$ a year due to the limited clinical, yet statistically significant, effectiveness. In the Spinraza infant onset type 1 SMA ENDEAR study, ${ }^{5}$ no infant who received sham therapy achieved any development milestone; how-

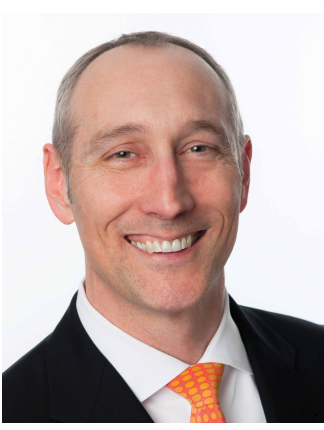
ever, 22\% of Spinraza-treated infants achieved head control, and $1 \%$ achieved standing with assistance. After 576 days of follow-up in the SHINE study, ${ }^{6}$ approximately $45 \%$ of treated infants achieved full head control, and 29\% achieved sitting independently. The median time to death or permanent ventilation was 22.6 weeks in the sham control group and 73.0 weeks in the Spinraza group. With Zolgensma, at 24 months, $92 \%$ of infants achieved head control, and $17 \%$ could walk independently. Among the 25 Zolgensma-treated patients, there less than list price. When the ICER cost-effective models are run for infants with SMA type 1 who are presymptomatic (i.e., at or near birth), the Zolgensma price at $\$ 100,000$ per QALY is $\$ 1.1$ million, 1.9-fold lower than list price. Spinraza is an intermittent intrathecal infusion with a wholesale acquisition cost of $\$ 805,000$ for the first year of therapy and $\$ 380,000$ per year thereafter. The Spinraza SMA type 1 presymptomatic costeffective modeling found a price of $\$ 72,800$ per QALY in year 1 and $\$ 36,400$ per QALY gained in year 2 and beyond, at the has been 1 nontreatment-related death and another potentially treatment-related death. ${ }^{7}$ Finally, the ICER analysis found higher gains in QALY and life-years gained for Zolgensma compared with Spinraza. ${ }^{1}$ Many of the additional Spinraza lifeyears gained could be spent requiring caregiving and medical care, resulting in the Spinraza maximum price of $\$ 36,400$ per year and the Zolgensma one-time price of \$1.1 million for SMA type 1 presymptomatic treatment. ${ }^{1}$

The ICER report helps ground the pricing conversation in facts, using comparability metrics (i.e., QALYs). Using these metrics, Spinraza is clearly overpriced compared with the value it brings, but using Zolgensma to treat 
presymptomatic infants may be more closely priced to the value (yet still 2 -fold too high). The ICER SMA report provides a "controversies and uncertainties" section, cataloging considerable fair pricing issues with Zolgensma. The issues include potential liver toxicity, extremely small number of patients studied without blinding or randomization, and limited follow-up. There are only 25 patients with high quality data-12 patients received Zolgensma at ages 0.9-7.9 months, with 38 months follow-up, and an additional 13 patients are still in clinical trial follow-up. All Zolgensma data are from single-arm, open-label study design, lacking randomization or a control group. It is unknown whether patients older than 8 months, more severely ill, or with a genetic profile different than those selected for the clinical trials will do as well. The small number of studied patients have had a median follow-up of 24 months. Zolgensma long-term durability, as well as safety, is unknown.

The hope is that Zolgensma will provide lifelong benefit to patients; however, if the gene expression wanes over time, the subsequent treatment pathway is unclear. If patients develop anti-adeno-associated virus antibodies to the Zolgensma gene delivery vector virus, they would be unable to receive another Zolgensma dose and likely unable to receive any other genebased therapy using an adeno-associated virus delivery mechanism. The ICER report nicely summarizes this as follows: "The current limitations of the clinical evidence for Spinraza and Zolgensma include study populations that limit the generalizability of clinical outcomes to SMA patients who differ from those included in clinical trials, limited long-term safety (e.g., repeated lumbar puncture procedures) and efficacy data (e.g., durability of novel gene therapy), and the uncontrolled, open-label Zolgensma study design." Finally, it is important to note that the most commonly cited ICER cost-effective models are optimistic regarding Zolgensma durability, assuming no patients died and no patients transitioned to ventilation.

ICER also created a symptomatic infant SMA type 1 pessimistic scenario model which assumed that $30 \%$ of patients in the "sitting" health state lose milestones, with a subset transitioning to ventilation and subsequent death and lower utilities. The Zolgensma pessimistic model results in a $46 \%$ decrease in QALYs gained. Using the 46\% QALY reduction, we estimate a corresponding Zolgensma price of approximately $\$ 185,000$ at the $\$ 100,000$ per QALY investment. Using the pessimistic and optimistic ICER models, we now have a range of Zolgensma fair pricing points for an investment of $\$ 100,000$ per QALY gained to treat symptomatic infants with SMA type 1 - $\$ 185,000-\$ 310,000$ — substantially lower than the $\$ 2.125$ million list price.

Some will defend that Zolgensma pricing should be set at potential Spinraza cost avoidance. However, it is counterintuitive to base Zolgensma pricing on Spinraza, which did not meet cost-effectiveness thresholds for any SMA population, and the current cost would have to be reduced 10 -fold to achieve
$\$ 100,000$ per QALY gained when treating an infant with presymptomatic SMA type 1, where cost-effectiveness was determined to be best. It is absurd to use an overpriced product for new therapy pricing points.

In addition, there are limited data on real-world use of Spinraza, and speculations are that patients do not remain on therapy as indicated in prescribing information. In a study presented at the 2019 Academy of Managed Care Pharmacy Annual Meeting, ${ }^{8}$ researchers found that Spinraza patients may not get all prescribed doses in their first year of therapy. It is important to note that the results are limited due to lack of data for patients obtaining patient assistance, patients in early access programs, and patients switching insurers (e.g., commercial plan to Medicaid). The Spinraza excessive price to value provided and potential for early therapy discontinuation constitute a strong case for manufacturer and payer valuebased contracting to recoup cost for Spinraza drug therapy failure that can be defined as early discontinuation or failure to obtain milestones, including ventilation. In addition, some insurers are requiring Spinraza discontinuation when Zolgensma therapy is administered.

For Zolgensma, the 1-time therapy and fixed 1-time cost presents unique challenges. AveXis, a Novartis company, has partnered with Accredo to offer a pay-over-time option of up to 5 years to help ease possible short-term budget constraints, especially for states, small payers, and self-insured employers. ${ }^{9}$ Value-based contracts are an essential component of the reimbursement model for high-cost gene therapies such as Zolgensma. However, there are several limitations and considerations to be discussed before a contract can be signed. Defining the outcome terms for recouping the upfront payment or when installment payment would stop requires highly specific data and patient tracking. For example, if ventilation or loss of milestones is a trigger outcome, then patient tracking and documentation will be required. If Spinraza is initiated after Zolgensma, does the contract allow for recouping Spinraza cost for as long as the patient requires Spinraza therapy? What if the patient is lost to follow-up? A patient may not want to be tracked or may switch health plans from where the original contract was signed and drug was paid. In addition, if a value-based contract includes a large discount or rebate for individuals who are considered treatment failures, the contract could set a new Medicaid lowest best price, thereby increasing the rebate paid to all state Medicaid agencies at the time of Zolgensma purchase.

The challenge brought by these 2 SMA therapies is continued health care affordability. The gene therapy pipeline is long, and the conversation about drug prices is not over. Is it sustainable that every rare condition therapy comes to market at Spinraza or Zolgensma pricing? ICER provides essential information to establish a fair price for the value that a drug provides. Hopefully, more data will continue to emerge on effectiveness and durability of 1-time novel gene therapies. 
Now is the time to determine the optimal approach for payment and management instead of letting this SMA gene therapy set a bar.

\section{Authors}

CATHERINE I. STARNER, PharmD, and PATRICK P. GLEASON, PharmD, Prime Therapeutics, Eagan, Minnesota.

AUTHOR CORRESPONDENCE: Catherine I. Starner, PharmD, Prime Therapeutics, 2900 Ames Crossing Rd., Eagan, MN 55121. Tel.: 800.858.0723, ext. 5073; E-mail: cstarner@primetherapeutics.com.

\section{DISCLOSURES}

No funding supported the writing of this commentary. The authors are employed by Prime Therapeutics, a pharmacy benefits management company.

\section{REFERENCES}

1. Institute for Clinical and Economic Review. Spinraza and Zolgensma for spinal muscular atrophy: effectiveness and value. Final evidence report. April 3, 2019 (updated May 24, 2019). Available at: https://icer-review.org/ wp-content/uploads/2018/07/ICER_SMA_Final_Evidence_Report_052419. pdf. Accessed October 19, 2019.

2. Spinraza (nusinersen) injection, for intrathecal use. Biogen. Revised June 2019. Available at: https://www.spinraza.com/content/dam/commercial/specialty/spinraza/caregiver/en_us/pdf/spinraza-prescribing-information.pdf. Accessed October 19, 2019.
3. Zolgensma (onasemnogene abeparvovec-xioi) suspension for intravenous infusion. AveXis. 2019. Available at: https://www.avexis.com/content/pdf/ prescribing_information.pdf. Accessed October 19, 2019.

4. Cohen J. At over $\$ 2$ million Zolgensma is the world's most expensive therapy, yet relatively cost-effective. Forbes. June 5, 2019. Available at: https://www.forbes.com/sites/joshuacohen/2019/06/05/at-over-2-millionzolgensma-is-the-worlds-most-expensive-therapy-yet-relatively-costeffective/\#2a93c05f45f5. Accessed October 19, 2019.

5. Finkel RS, Mercuri E, Darras BT, et al. Nusinersen versus sham control in infantile-onset spinal muscular atrophy. New Engl J Med. 2017;377(18):1723-32.

6. Castro D, Farrar M, Finkel R, et al. P.170 Interim report on the safety and efficacy of longer-term treatment with nusinersen in infantile-onset spinal muscular atrophy: results from the SHINE study [abstract]. Neuromuscular Disorders. 2018;28(Suppl 2):S79-S80.

7. Terry M. A 2nd patient death reported in Novartis' gene therapy trial. BioSpace. April 22, 2019. Available at: https://www.biospace.com/article/a2nd-patient-death-reported-in-novartis-gene-therapy-trial/. Accessed October 19, 2019

8. Starner C, Gleason P. Spinal muscular atrophy: an integrated medical and pharmacy claims analysis of nusinersen uptake and gene therapy forecast among 15 million commercially insured [abstract]. J Manag Care Pharm. 2019:25(3-a):S54. Available at: https://www.jmcp.org/doi/pdf/10.18553/ jmcp.2019.25.issue-3-a

9. Novartis. AveXis announces innovative Zolgensma gene therapy access programs for US payers and families. May 24, 2019. Available at: https:// www.novartis.com/news/media-releases/avexis-announces-innovativezolgensma-gene-therapy-access-programs-us-payers-and-families. Accessed October 19, 2019 